\title{
The production of pigments \& hydrogen through a Spirogyra sp. biorefinery
}

\author{
R. Pacheco ${ }^{a}$, A.F. Ferreira ${ }^{a, *}$, T. Pinto ${ }^{b}$, B.P. Nobre ${ }^{b}$, D. Loureiro ${ }^{c}$, P. Moura ${ }^{\text {b }}$, L. Gouveia ${ }^{\text {b }}$, C.M. Silva ${ }^{\text {a }}$ \\ a IDMEC/LAETA - Instituto Superior Técnico, Universidade de Lisboa, Av. Rovisco Pais, 1, 1049-001 Lisbon, Portugal \\ ${ }^{\mathrm{b}}$ LNEG - Laboratório Nacional de Energia e Geologia, Unidade de bioenergia, Estrada do Paço do Lumiar 22, 1649-038 Lisbon, Portugal \\ ${ }^{\mathrm{c}}$ LNEG - Laboratório Nacional de Energia e Geologia, Unidade de energia Solar, Estrada do Paço do Lumiar 22, 1649-038 Lisbon, Portugal
}

\section{A R T I C L E I N F O}

Article history:

Received 16 September 2014

Accepted 19 October 2014

\section{Keywords:}

Spirogyra sp.

Electrocoagulation

Solar drying

Biohydrogen

Pigments

Life cycle assessment

\begin{abstract}
A B S T R A C T
This paper discusses the overall energy consumption and greenhouse gas emissions when extracting pigments and producing hydrogen from Spirogyra sp. microalga biomass. The energy evaluation from the biomass leftovers was also included in this work. The influence of the functional unit and different allocation criteria on the biorefinery assessments is also shown. The study consists of laboratory tests showing Spirogyra sp. growth, harvesting, drying, pigment extraction and fermentation by Clostridium butyricum. Electrocoagulation and solar drying were tested and compared to conventional centrifugation and electrical dewatering in terms of their energy consumption for harvesting and dewatering, respectively. To discuss the biorefinery viability, the pigments and biohydrogen $\left(\mathrm{bioH}_{2}\right)$ retail costs are considered against operational costs according to electricity needs. The low yield of biochemical hydrogen and the high energy requirements for the pigment extraction were identified as main topics for further research. This research hopefully contributes to highlight the importance of energy and emission balances in order to decide on feasibility of the biorefinery.
\end{abstract}

(c) 2014 Elsevier Ltd. All rights reserved.

\section{Introduction}

Recently, microalgae are receiving a considerable attention due to their ability to synthesize valuable compounds (e.g. pigments) accumulate high energy compounds (e.g. lipids, carbohydrates) and sequester carbon. They are therefore considered as a third generation feedstock for biofuel production and have a great potential as renewable feedstock [1-3]. Exhaustive studies have been done on the production of biofuels through biological and thermochemical methods using microalgae. Microalgae biomass can also be used as the feedstock for hydrogen production by dark fermentation [4-6]. For this process it is advantageous that the microalgae have the ability to accumulate a high content of storage carbohydrates. However, as biofuel-based microalgae remains expensive, the biorefinery is a way to overcome this bottleneck, taking advantage of all biomass components (e.g., Nobre et al. [7]; Ferreira et al. [8]; Demirbas [9]).

Spirogyra sp. is a filamentous green alga, which is common in freshwater habitats. It contains about $11-21 \%$ of lipids and a high

* Corresponding author at: IDMEC/LAETA - Instituto Superior Técnico, Dept. of Mechanical Engineering, Av. Rovisco Pais, Pav. Mecânica I, 2 Andar, 1049-001 Lisbon, Portugal. Tel.: +351 218419546; fax: +351218417640.

E-mail address: filipa.ferreira@tecnico.ulisboa.pt (A.F. Ferreira). content of sugar, about 33-64\% [10]. Spirogyra sp. contains Chlorophyll $a$ and Chlorophyll $b$ which are responsible for its green color. However, in some culture/stress conditions the microalga appears yellow or orange due to the presence of secondary pigments (carotenoids). These carotenoids are of high value, for example the astaxanthin is recognized as a potential anti-oxidant, and could prevent cancer and cardio-vascular problems [11,12]. The high productivity of the microalga Spirogyra sp. and its capacity to accumulate high amounts of sugar, make this biomass also attractive as substrate for bioethanol and bioH $\mathrm{b}_{2}$ production. The biofuel production by this microalga is still in development and so far, there are no references in the literature related to $\mathrm{bioH}_{2}$ production from Spirogyra sp. biomass. Nevertheless, several studies, regarding bioH $_{2}$ production using other microalgae, have already been published (Table 1 ).

As it can be seen in Table 1, centrifugation is one of the most frequently used harvesting methods for microalgae biomass. The process uses centrifugal forces to separate solids in suspension from the liquid. Although centrifugation is an effective harvesting method, it presents high investment and operational costs. The electrocoagulation method was studied in detail for Nannochloropsis sp. [13].

The authors obtained the best recovery (>97\%) using a current density of $8.3 \mathrm{~mA} \mathrm{~cm}^{-2}$ for $10 \mathrm{~min}$ without significantly changes 